\title{
Method for Eliciting and Analyzing Business Processes Based on Storytelling Theory
}

\author{
Pedro Antunes \\ Victoria University of Wellington \\ pedro.antunes@vuw.ac.nz
}

\author{
Jose A. Pino \\ DCC - University of Chile \\ jpino@dcc.uchile.cl
}

\author{
Mary Tate \\ Queensland University of Technology \\ mary.tate@qut.edu.au
}

\begin{abstract}
In this paper we suggest that storytelling theory can be used to analyze business processes in two behavioral dimensions, model and context, which concern predefined and improvised behavior, respectively. We develop a method to elicit stories about business processes from process participants. By applying the method in two cases, we provide some evidence on what type of analysis can be done and how it can impact the design of business processes. This research contributes with an innovative method to analyze integrated context/model behavior in business processes.
\end{abstract}

\section{Introduction}

We can regard organizations as entities that execute activities (A) in the scope of business processes (P), which are purposefully designed (D) to produce value (V) for stakeholders. When designing a business process, an organization has to decide how to apply technology (T) and humans $(\mathrm{H})$ across the various activities that set up the process. Even though the human and technology components can contribute with knowledge and information processing capabilities required to implement business processes, they do it in very different ways. Technology operates according to models (M), i.e. predefined routines and rules, while humans operate based on context (C), i.e. contingency and emergence. We may therefore suggest that process design actually involves making decisions regarding how to integrate context/model behavior.

This view, which we delineate in Fig. 1, is of course a very simplified account of reality. For instance, it ignores the role of technology in decision making, cognition, communication, and human augmentation. However, we find it useful to accentuate the need for designers to understand integrated context/model behavior in business processes, which is the main challenge addressed by this research.

In a broad perspective, this problem resonates with a long tradition of research about the relationships

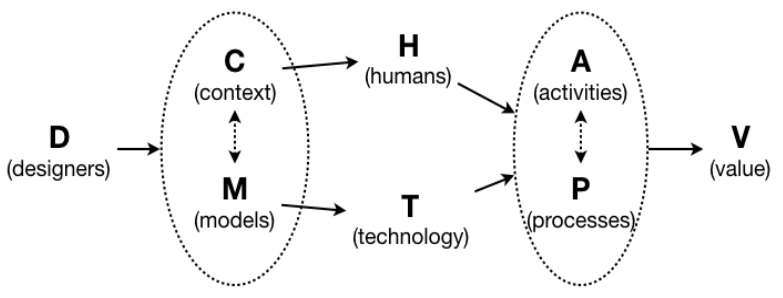

Fig. 1. The integration of context and models in business processes

between the social and technological facets of organizations, which we can find in preeminent theories developed in the social sciences such as actor-network theory [13], situated action [46] and frames theory [32]. It also resonates with notable research in computer science, which has been developing techniques to increase system flexibility [37], coordination [12] and context support $[22,23]$.

The field of design science research is differentiated from social sciences and computer science [21]. It is centered on the design of information systems artifacts. Many artifacts depend on theory, models and systems brought to us by computer science, while being scaffolded by theory, models and methods brought to us by social sciences [20]. Our research perspective concerns designers using analytic methods to explore integrated context/model behavior in business process artifacts, which aligns with design science by regarding methods as primary information systems artifacts [8].

Here we propose a method to analyze integrated context/model behavior using storytelling theory.

We apply the method in two cases and reflect on the results. The major contribution of this study is the innovative use of storytelling theory in the analysis of business processes.

The paper is structured as follows. Section 2 presents the research framework, concerning the integration between model-based and context-based behavior in organizations. In Section 3 we ground the research on theory and methodology. In particular, we adopt storytelling theory as the conceptual foundation for the research. Section 4 presents the method, which has been divided in three parts concerning the data collection instrument, data collection procedure, and data analysis 


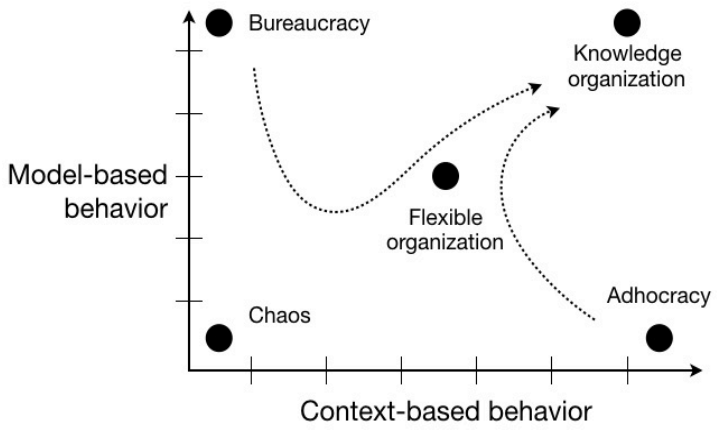

Fig. 2. Integration between model-based and context-based behavior

procedure. In Section 5 we justify the method by applying it in two cases. In Section 6 we focus on the implications of the research for the development of knowledge organizations. Finally, in section 7 we present some concluding remarks.

\section{Research Framework}

Organizations can be conceived in two dimensions, one related to model-based behavior and another related to context-based behavior (Fig. 2). The first dimension considers the extent of procedural knowledge (routines and rules [5]) in process execution. Model-based behavior may range between a coarse account of responsibilities, which can be used to structure work but not to control, and a detailed, step-by-step account of actions, which are amenable to benchmarking, computer control and automation [15].

The second dimension considers the extent of contextual knowledge supporting contingent, emergent and improvised behavior [25]. Context is an overarching concept that can be used to characterize any situational change [14], which may be caused by internal and external factors [48]. As internal factors, we consider any contingencies affecting process execution, such as time pressure, deviations, suspensions, omissions, mishaps, reduced performance, and individual capabilities [1, 40, 42], as well as the practices developed by organizations to tackle them, e.g. trough team work and standardization [48]. Regarding external factors, we consider any external events affecting the execution environment, such as turbulence, legislation and competition [35].

Organizations can then be positioned in the abovementioned dimensions according to how they engage in model-based and context-based behaviors. If they engage exclusively in model-based behavior, that means they have well-defined routines, reduced communication, and fine-grained control. They can as well be regarded as administrative bureaucracies [6]. If organizations engage exclusively in context-based behavior, then they can be classified as adhocracies. Adhocracies are characterized by operating in dynamic, improvised and emergent scenarios, privileging collaboration over planned coordination [29]. A lack of engagement in both model-based and context-based behavior can be regarded as chaos [45]. In such organizations, behavior is erratic, communication is unconnected, and knowledge is under-utilized [44].

Of course, bureaucracy, adhocracy and chaos are just archetypes of extreme behavior. It seems reasonable to suggest that most organizations prefer to avoid these archetypes and instead strive for integrated model-based and context-based behavior. We can then identify two new archetypes: the flexible organization and the knowledge organization (Fig. 2). The flexible organization is fundamentally model-driven, but designed with routines and rules that can accommodate variability, looseness, adaptation and evolution [37]. This can be accomplished by generalizing routines and introducing rules to deal with unexpected events [24], exceptions [2, 27], unique cases [31], and emergent patterns [9], while at the same time increasing the reach of context-based behavior, which is necessary to operate outside the procedural envelope [3].

The knowledge organization goes beyond the flexible organization by bolstering the benefits of integrated model/context behavior. This type of organization is capable to utilize the technological infrastructure to support procedures and control while at the same time supporting tacit and strategic changes [11].

As shown in Fig. 2, organizations move away from bureaucracy, adhocracy and chaos towards flexible organizations by removing constraints imposed by model and context; and then evolve towards knowledge organizations by fully integrating model and context. As suggested by research, contemporary organizations are compelled to take this journey because they are facing more interactive and dynamic business environments $[22,23,33,39]$.

The integration of model-based and context-based behaviors can be either static or dynamic [22]. Static integration extends models with variables affecting processes [39]. Examples include models that use context variables such as location, time and resources [41]. Dynamic integration considers the interactional [23] and performative [22] facets of the process. The interactional facet recognizes that actions performed by actors involved in business activities can cause opportunistic contextual changes, while the performative facet addresses issues such as local availability of time, effort and expertise, which can only be committed at the exact time they are required [38]. In this research, we address the interactional and 
performative facets of business processes by analyzing how process participants see themselves participating in the processes, rather than just looking at the models governing the processes.

\section{Theory and Methodology}

This research uses storytelling theory [7, 10]. Storytelling stems from the area of narrative studies, and seeks to explain how humans make sense of their experiences by telling stories [17]. The theory has been transferred to the organizational domain with the purpose of explaining how people in organizations use stories to define structure, make commitments and exert control $[10,36]$. We find exactly the same goals in model-based behavior, even though the paradigms are different: storytelling uses narrative while models use specialized constructs and notations [34].

A story is an account of an event or series of events, which are usually enriched with contextual knowledge about what happened, who was involved, where it happened, and so forth. The common features of stories and models are that both may describe interrelated activities, along with the notions of order, communication, execution, control, responsibility, and decision [47]. However, stories and models also exhibit some fundamental differences. Models formalize routines, rules and relationships, while stories embed that procedural knowledge with other contextual elements using narrative. Furthermore, models are usually defined to avoid ambiguity between who defines and uses them, while stories can be interpreted in many meaningful ways. That is, stories are more informal, eventually richer, accounts of events than models.

In summary, we can say that storytelling theory integrates elements of model-based behavior with elements of context-based behavior appearing in stories. The value brought by storytelling theory to our research is regarding both model and context as an integral part of a story.

\subsection{Methodology}

Our research adopts storytelling theory for analytical purposes, using a comparative approach. Many comparative studies in the qualitative tradition are designed to compare two groups (A and $\mathrm{B}$ ) taking different treatments (A-B design) [26]. Another popular approach compares two observations from the same group $(A)$ before and after applying a treatment $\left(A_{t 0}-A_{t 1}\right.$ design). Both these approaches provide strong baselines for comparative analysis. However, we also find approaches where a baseline group (A) is compared against an aggregate of multiple observations $\left(A-B_{0-n}\right.$ design), and also against the individual observations (A$\mathrm{B}_{0} \ldots \mathrm{B}_{\mathrm{n}}$ design) [19]. The former approach allows to qualitatively appraise the strength and consistency of observed patterns, while the latter approach emphasizes the significance of outliers, variations and unique observations.

The method reported in this paper adopts a variation of the two latter approaches. In our method, what we designate as group $\mathrm{A}$ is a collection of models describing official processes. An official process defines and standardizes organizational behavior, is well-known by the members of the organization, is instantiated repeatedly and frequently, and is formalized in official documentation.

What we designate as group B is a collection of stories about official processes reported by process participants. Thus, group B contains data about modelbased and context-based behaviors. We use these stories in aggregate form (noted earlier as $\mathrm{B}_{0-\mathrm{n}}$ ) to analyze the strength and consistency of identified patterns, but also analyze individual stories (noted earlier as $\mathrm{B}_{0} \ldots \mathrm{B}_{\mathrm{n}}$ ) to identify unique and extreme cases.

Following the qualitative research tradition, the collected stories are analyzed in multiple rounds using coding techniques [30]. Qualitative studies have been using two different coding strategies: inductive and deductive [16]. The inductive strategy uses open coding, giving the researcher freedom to create categories for any element in a story that may seem interesting. Deductive coding restricts the researcher to use predefined categories, even though the categorization can be iteratively improved. For this research, we adopted the deductive approach. One reason for this decision is to increase reliability and ease of use. Furthermore, the existing literature already provides a reasonable collection of categories that can be reused $[22,43]$.

\section{Method \\ 4.1. Data collection instrument}

The data collection instrument adopts the suggestion by [4] to elicit stories about business processes using cartoons. We use a slide presentation template that can be edited by off-the-shell tools such as Apple's Keynote, Microsoft's PowerPoint and Google's Slides. The template invites participants to tell a story using the slide presentation. The template can be downloaded from the first author's website.

The template contains a collection of master slides, each one showing a cartoon depicting a common business situation (Fig. 3). Examples include writing a document, having a meeting, making a phone call, and sending a message through the computer. The cartoons are generic and can be used to describe a wide range of 


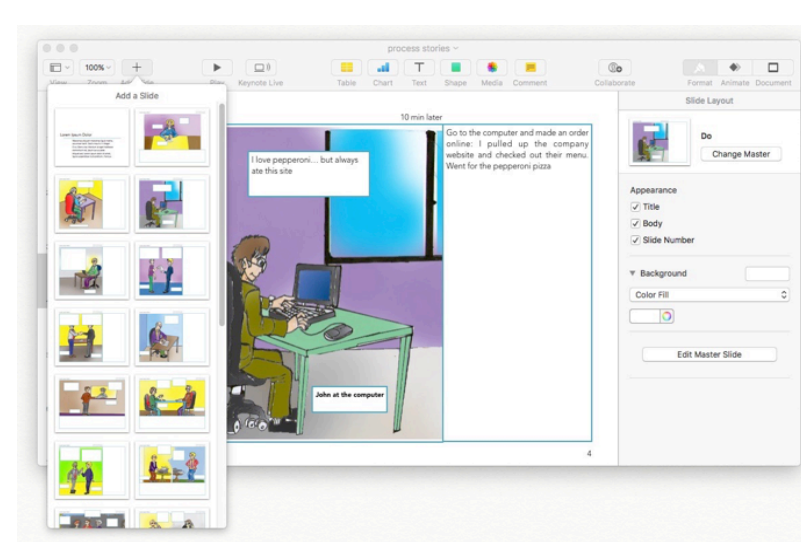

Fig. 3. Slide template for telling stories

business situations. The cartoons can have several characters and business objects. For instance, there is a cartoon showing a character writing a document, while another shows a conversation between two characters.

When telling stories, participants can pick up different master slides and configure a sequence of predefined visual and textual elements. In particular, above each cartoon, two text boxes help summarizing the situation (e.g. "ordering product") and defining time references (e.g. "the next day" and "30 minutes after"). These specific uses are not mandatory but instead suggested by the template.

Narrative boxes are available next to cartoons (either below or to the side), so that participants can provide a narrative account of the depicted activities, events and any other contextual elements they wish to report (e.g. to describe what was discussed in a meeting or why a meeting was postponed). Dialogue bubbles can also be used to put words in the mouth of characters. Label boxes can be used to name characters and objects.

\subsection{Data collection procedure}

Data collection starts by emailing the template to the participants and asking them to describe a known process. The template contains an example story and a slide with a short message inviting the participants to tell a story (about the process) using the same approach. The participants then tell their stories by picking and configuring the slides. In the email, we advise the participants that the template has a collection of configurable master slides, but do not provide specific instructions on how to tell stories. The participants send back their presentations through email.

After receiving the slide presentations, the experimenter removes the example stories from the documents, and the documents are printed to pdf. A pdf editor can then be used to analyze the stories.

\subsection{Data analysis procedure}

Data analysis starts by coding the stories using the following codes:

Model-based elements

- Activities: What actions are performed within the process scope

- Decisions: Decisions made in the process

- Actors: People conducting activities

Context-based elements

- People: References to people not conducting activities

- Emotional context: Expressions of mental states, such as stress, irritation and disbelief

- Settings: References to specific work settings required by the process, including in particular the use of computing tools and applications

- Interactional context: References to interactions and collaborations between actors and people

- Decisional context: References to individual factors affecting a decision, which in particular may include explanations about why a certain decision is made or a certain process activity is performed

- Environmental context: Accounts of uncontrolled events and constraints, which affect the process but are not determined by the actors in the process

- Locations: References to physical locations

- Time: References to time, including urgency, deadlines, time constraints, and frequency of occurrence

- Methods: References to ways of doing, which reflect the patterned characteristics of processes

One concern we found when using these codes is that we have to avoid methods being coded as decisions and vice versa. To avoid the problem, we define the rule that the "method" code should reflect an organizational viewpoint (what the organization suggests actors should do), while the "decision" code should be exclusively used when the story conveys an individual point of view (what an actor decides to do).

Another rule concerns the possible intricacies between decisions and emotions. While a story may present a decision along with an emotion, we establish the rule that the "emotion" code should only be used when the feeling (e.g. satisfaction, angst) is not the single determinant of a decision.

We also set a rule helping to decide what to do with repeated elements appearing in stories. A typical example occurs with the time element, e.g. when a story refers multiple times that something happens by the end of the week. Another example is when the author has copied and pasted the same element across different slides, e.g. indicating that someone was working with 
the computer or located in a specific place. On the other hand, in some stories, it seems clear that the storyteller wanted to emphasize a continuing situation, e.g. highlighting that someone waited for something to happen for a long time. Thus, we set the rule that coding should reflect the actual intent of the storyteller: a repeated element should be counted multiple times if repetition reflects a decision made by the storyteller, using that particular narrative style to emphasize a concept; and it should be counted just once if repetition is the mere consequence of using cut and paste in the story composition.

We also found that sometimes participants report several emotions together, in the same slide and occasionally even in the same phrase. The set rule is that multiple emotions appearing in the same narrative box should be taken as a whole and counted only once, but if appearing in different places in the same slide, they should be counted separately. Once again, the rule aims to adhere to the storyteller' intents.

We have been coding stories using two coders. Coders individually analyze the stories and then get together to discuss doubts, identify conflicts, and to reach consensus. Using a consensual approach may increase the quality of the results, as found in software review meetings [28].

After coding, the procedure continues with counting the number of elements per category. Averages and standard deviations can then be used to analyze the strength of each category. Whenever necessary, we go back to the stories to qualitatively analyze the data. We use the Mann-Withney test at 0.05 significance to analyze if the results from two different processes can be aggregated; and also, to compare two different categories. Unique occurrences in each individual story are also analyzed to reveal distinctive aspects of a process. After coding and categorization, we can finally analyze in detail the integrated context/model behaviors. The method is further described along with two cases presented in the next section.

\section{Cases}

\subsection{Processes and participants}

The organizations selected for this study were university departments located in different countries and with different cultural and educational practices (a computer science department and a business school). For the purposes of this research, the two universities should be regarded as any other professional organization.

The official processes were carefully selected. They are representative of core business activities performed by the universities. They are formalized in official

\section{Table 1. Participants (NOTE: One GP participant generated 2 stories)}

\begin{tabular}{l|l}
\hline HEC & GP \\
\hline $\begin{array}{l}\text { 2 staff applicants (1 female } \\
\text { and 1 male) }\end{array}$ & $\begin{array}{l}5 \text { supervisors (1 female and 4 } \\
\text { male) }\end{array}$ \\
\hline $\begin{array}{l}5 \text { student applicants (3 } \\
\text { female and 2 male) }\end{array}$ & $\begin{array}{l}5 \text { students (1 female and } 4 \\
\text { male) }\end{array}$ \\
\hline 1 researcher (male) & - \\
\hline & 1 administrator (female) \\
\hline 8 participants & 11 participants \\
\hline
\end{tabular}

documents, executed repeatedly and frequently and enforced to the participants.

One official process implements a university-wide policy regarding ethics in research data collection, which is known internally as Human-Ethics Committee (HEC). Every research project done in the university and involving human participants must be evaluated by a human ethics committee, which assesses the research objectives, research design, data collection procedures, selection of participants, etc. The HEC process deals with research project applications and is fully implemented online (person-to-application [47]).

The HEC process is well known within the university since researchers (students or not) often submit projects many times a year. It is carefully and extensively documented. Besides the university policy, several documents explain how applications flow between applicants, administrative staff, committee members, and head of HEC. One document in particular includes a model (using flowcharts) describing the model-based behavior, which is also enforced by the online system. We used that model in our study.

The second official process deals with the completion of the Graduation Project (GP), which is the final undertaking of an engineering degree. The process takes a year to complete, from initial proposal to examination, and involves every student enrolled in the final year of studies. The process is not fully implemented online, but at some points requires using a system (i.e. predominantly person-to-person [47]).

The GP process is publicly described to the student cohort once a year. It is also documented in two slide presentations available to students and supervisors. Two models (using activity diagrams) are included in the slides that describe the GP process in two stages, the first one dealing with topic selection and the other dealing with project completion. We used these two models in our study. The HEC and GP models define what we designated as group A.

Regarding group B, we recruited participants with significant experience with the HEC and GP processes. In the HEC case, we recruited participants among staff, students and invited researchers that had previously completed a project submission with success. Having succeeded as an applicant was essential to make sure the 
participants knew well the process. For the GP case, the participants were selected among supervisors and students that had successfully completed graduation projects. We also collected data from the administrator that is responsible for ensuring that supervisors and students complete the process according to the set rules and timelines. Table 1 summarizes the main characteristics of the participants.

\subsection{Case results}

In order to provide a wide-ranging view of the results, we cycle the analysis between quantitative and qualitative evidence. Quantitative evidence is summarized in Table 2. Qualitative evidence is restricted because of space limitations.

All stories created by the participants contained rich knowledge about the official processes, combining both model-based and context-based elements. For every story we could recreate the underlying official process, although with different granularity and focus. Variations in number of activities, actors and decisions illustrate the point. Activities ranged between 7 and 37 for GP, and 5 and 23 for HEC.

Stories with fewer activities usually shortened parts of the official process using umbrella activities such as "decide topic" (GP8) or "fill out form" (HEC5). This shortening reflected more a pragmatic than an abstract view of the process. However, even these brief stories contained very relevant contextual knowledge about official processes. For instance, GP8 mentioned twice the lack of information necessary to decide a topic, which highlights an important weakness in the official process: "[I do] not know the project requirements, because there is no official information available", and later, "[I have] to select a topic but [do] not know which to select, because [I do] not know what is required". Regarding HEC5, even though few details about the "fill out form" activity were provided, the story was vivid about how the official process failed after that step: "I hear nothing", "I haven't heard anything about my application", and "still hear nothing".

Stories with most activities, such as GP12 and HEC2, contained many model-based elements missing in official processes, complemented by rich contextual knowledge. For instance, HEC2 provided details about a failed submission: "what? So quick? No no no... this is not right...", "why [a reply] from [person], did I fill up the wrong name?" and "I revised my application".

In Table 2, we report averages and standard deviations for each element analyzed in stories. We also show the results from the Mann-Withney test (p-value). As already noted, the purpose of the Mann-Withney test was to check if the data from the two cases could be aggregated or not. For all contextual elements except one, the Mann-Withney test indicated that the hypothesis that the two samples have the same means cannot be rejected. We used these results to support the decision to aggregate the two cases when the MannWithney test was above 0.05. Setting was the only contextual category with a result below 0.05 . This result can be explained by the different characteristics of the GP and HEC processes: GP is mainly person-to-person, while HEC is mainly person-to-application. It is therefore understandable that HEC has more setting elements than GP. Considering these results, we removed the setting category from further analysis.

On average, the total number of elements in stories was high: each participant reported 67.65 elements per story. This number seems especially relevant when we consider the actual sizes of the official processes on which the stories were based: the official processes had an average size of 18 elements (10.5 activities, 4 actors and 3.5 decisions). This also highlights that the ratio between stories and official processes was 3.76; and the ratio between context and model elements in stories was 2.25 . Roughly speaking, for each element in the official process, we captured almost four elements using stories, of which more than two concerned context-based behavior.

The distribution of contextual elements per story was the following: $23.39 \%$ time; $20.49 \%$ people; $15.70 \%$ decisional; $13.25 \%$ interactional; $12.58 \%$ method; and $7.24 \%$ emotional. It was a surprise that the majority of reported contextual knowledge was related to time. Time not only relates to when events occur but also other aspects such as waiting for events or accentuating the passage of time. Many of the time codes in HEC regarded waiting (e.g. "waiting patiently", "you will get some feedback soon", and "waiting for so long"), and lack of feedback (e.g. "after submission, nothing happened", and "I hear nothing"). In GP, time was more linked to the natural duration of activities (e.g. "in the first weeks", "after a week", and "after a short time"). These results clearly suggest that the time element may help uncovering problems with model-based behavior.

The number of contextual elements related to people was also interestingly high. The participants often referred to stakeholders indirectly related to the process but not explicitly referred to in the official process. These results suggest that model-based behavior may excessively emphasize who does what, instead of including other, perhaps more diffuse, types of relationships.

The number of elements related to decisional context was higher than the number of elements related to method (7.05 versus 5.65, respectively). Applying the Mann-Whitney test to the two samples showed the differences were significant (p-value 0.03752). Indeed, 
Table 2. Results for the two cases. The last lines show p-values from the Mann-Withney test, and averages and standard deviations (NOTES: GP3 and GP9 are from the same participant. GP12 is from the administrator).

\begin{tabular}{|c|c|c|c|c|c|c|c|c|c|c|c|c|c|}
\hline & \multicolumn{12}{|c|}{ GP process } & \multirow[b]{3}{*}{ Total } \\
\hline & \multicolumn{3}{|c|}{ Mode-based knowledge } & \multicolumn{9}{|c|}{ Context-based knowledge } & \\
\hline & Activities & Actors & Decisions & People & Emotional & Setting & Interactional & Decisional & Environment & Location & Time & Method & \\
\hline GP1 & 17 & 3 & 3 & 9 & 2 & 0 & 4 & 7 & 0 & 0 & 9 & 1 & 55 \\
\hline GP2 & 13 & 3 & 2 & 5 & 1 & 0 & 4 & 6 & 0 & 0 & 5 & 14 & 53 \\
\hline GP3 & 10 & 3 & 4 & 3 & 0 & 0 & 2 & 6 & 0 & 0 & 1 & 5 & 34 \\
\hline GP4 & 27 & 4 & 7 & 16 & 0 & 1 & 13 & 14 & 4 & 0 & 11 & 9 & 106 \\
\hline GP5 & 23 & 5 & 2 & 7 & 2 & 2 & 8 & 6 & 13 & 3 & 13 & 3 & 87 \\
\hline GP6 & 7 & 3 & 2 & 9 & 8 & 0 & 5 & 19 & 4 & 0 & 7 & 2 & 66 \\
\hline GP7 & 25 & 5 & 2 & 10 & 5 & 2 & 13 & 12 & 1 & 0 & 21 & 5 & 101 \\
\hline GP8 & 8 & 3 & 1 & 4 & 9 & 0 & 5 & 6 & 2 & 0 & 8 & 2 & 48 \\
\hline GP9 & 11 & 3 & 3 & 8 & 1 & 0 & 4 & 3 & 0 & 0 & 1 & 8 & 42 \\
\hline GP10 & 11 & 3 & 0 & 4 & 5 & 0 & 3 & 12 & 1 & 0 & 14 & 2 & 55 \\
\hline GP11 & 9 & 2 & 0 & 9 & 2 & 2 & 5 & 4 & 4 & 0 & 8 & 2 & 47 \\
\hline GP12 & 37 & 7 & 3 & 33 & 3 & 9 & 25 & 5 & 0 & 2 & 21 & 29 & 174 \\
\hline AV & 16.50 & 3.67 & 2.42 & 9.75 & 3.17 & 1.33 & 7.58 & 8.33 & 2.42 & 0.42 & 9.92 & 6.83 & 72.33 \\
\hline SD & 9.43 & 1.37 & 1.88 & 8.13 & 2.98 & 2.57 & 6.56 & 4.81 & 3.73 & 1.00 & 6.56 & 7.96 & 39.48 \\
\hline
\end{tabular}

\begin{tabular}{|c|c|c|c|c|c|c|c|c|c|c|c|c|c|}
\hline & \multicolumn{12}{|c|}{ HEC process } & \multirow[b]{3}{*}{ Total } \\
\hline & \multicolumn{3}{|c|}{ Mode-based knowledge } & \multicolumn{9}{|c|}{ Context-based knowledge } & \\
\hline & Activities & Actors & Decisions & People & Emotional & Setting & Interactional & Decisional & Environment & Location & Time & Method & \\
\hline HEC1 & 9 & 3 & 0 & 5 & 1 & 9 & 1 & 5 & 3 & 2 & 16 & 2 & 56 \\
\hline HEC2 & 23 & 4 & 0 & 16 & 7 & 11 & 7 & 6 & 3 & 1 & 22 & 6 & 106 \\
\hline HEC3 & 7 & 3 & 0 & 5 & 5 & 2 & 2 & 3 & 3 & 1 & 12 & 2 & 45 \\
\hline HEC4 & 23 & 6 & 1 & 14 & 2 & 5 & 6 & 9 & 9 & 0 & 4 & 5 & 84 \\
\hline HEC5 & 5 & 3 & 0 & 7 & 2 & 2 & 4 & 3 & 1 & 0 & 8 & 1 & 36 \\
\hline HEC6 & 7 & 6 & 0 & 8 & 7 & 5 & 4 & 4 & 4 & 0 & 19 & 5 & 69 \\
\hline HEC7 & 12 & 3 & 0 & 5 & 1 & 3 & 0 & 5 & 1 & 2 & 7 & 8 & 47 \\
\hline HEC8 & 9 & 4 & 0 & 7 & 2 & 3 & 4 & 6 & 1 & 1 & 3 & 2 & 42 \\
\hline AV & 11.88 & 4.00 & 0.13 & 8.38 & 3.38 & 5.00 & 3.50 & 5.13 & 3.13 & 0.88 & 11.38 & 3.88 & 60.63 \\
\hline SD & 7.16 & 1.31 & 0.35 & 4.27 & 2.56 & 3.34 & 2.39 & 1.96 & 2.64 & 0.83 & 7.05 & 2.47 & 24.14 \\
\hline p-value & 0.15 & 0.15 & 0.15 & 0.15 & 0.82 & 0.00 & 0.11 & 0.89 & 0.28 & 0.18 & 0.76 & 0.56 & 0.76 \\
\hline AV & 14.65 & 3.80 & 1.50 & 9.20 & 3.25 & & 5.95 & 7.05 & 2.70 & 0.60 & 10.50 & 5.65 & 67.65 \\
\hline SD & 8.71 & 1.32 & 1.85 & 6.74 & 2.75 & & 5.59 & 4.17 & 3.28 & 0.94 & 6.61 & 6.42 & 33.94 \\
\hline
\end{tabular}

in general the participants did not provide many procedural insights, instead describing what they decided to do at certain stages (e.g. "to clarify the topic, he has to read some papers"). This suggests that stories may be more adequate to capture concrete knowledge than models.

Fig. 4 shows the frequency distribution of contextual elements in the collected stories using a set of categories. The categories were selected to balance fragmentation and summation. The results show that the majority of stories contain a moderate (3 to 10) number of contextual elements referring to people, interactions, and decisions. On the other hand, the majority of stories contain a small ( 0 to 2 ) number of contextual references to methods and locations. Time seemed to diverge from these two groups, as a good number of stories contributed with a large number of time elements (more than 10). Environmental context also seemed to diverge from the two groups, showing a low to moderate occurrence of contextual references.
Looking into individual stories, GP12 stood out as a very significant outlier. To start with, this story had many more elements than the others (174 versus an average of 67.65). It also stood out by the number of contextual elements referring to people (33 versus an average of 9.20), method (29 versus an average of 5.65) and interaction (25 versus an average of 5.95). This story was generated by the GP process administrator. This could explain the unusual number of method elements, as the person has to frequently explain the process to others. However, it is also interesting to note the accumulated contextual knowledge about people and their interactions in this particular story. This not only reveals the author's particular expertise, but also the extent of her contextual knowledge. Notably, this was the only story documenting various ways to accomplish the process (e.g. "if the second revision is not accepted, they fail the course"). 


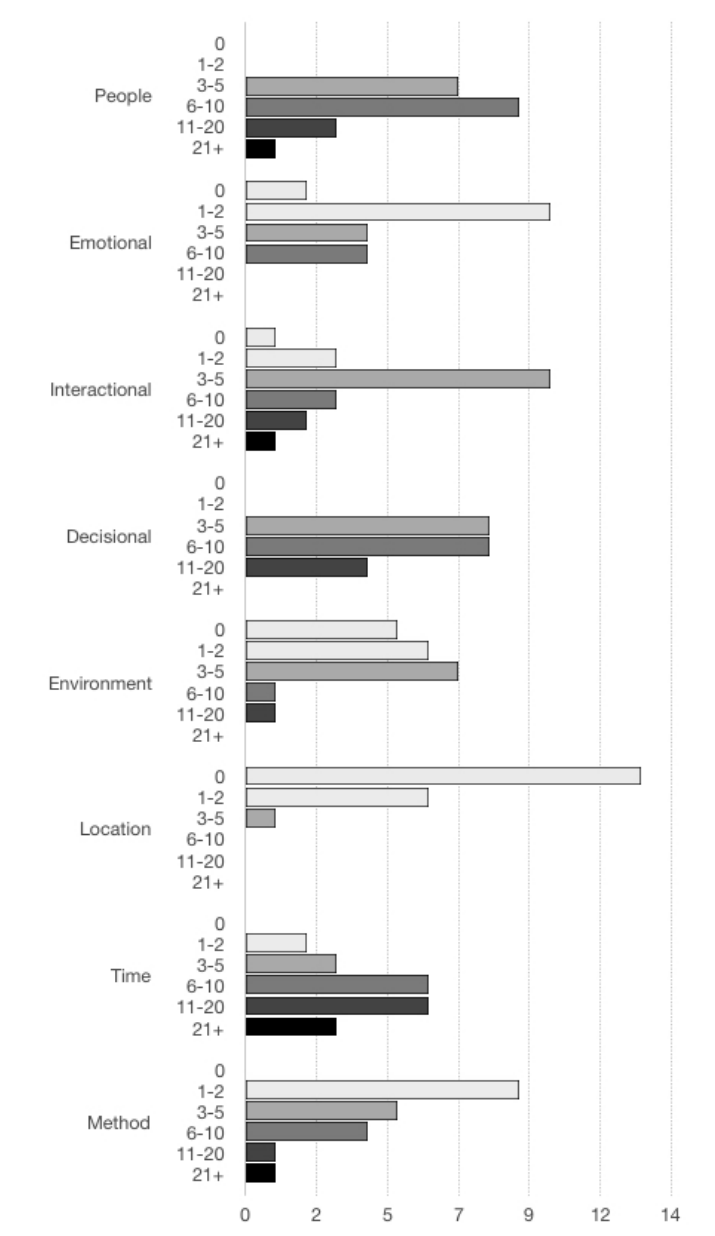

Fig. 4. Frequency distribution of contextual elements

\section{Method Assessment/Justification}

We now use the two cases to assess the contribution of the method to the research goals, i.e. the analysis of integrated context/model behavior using storytelling theory. The first consideration to make is about the extent and range of extracted knowledge. As previously noted, we gathered an average of 67.65 elements per story. A comparison with the elements in official processes, gives a ratio of 3.76. A comparison between the model-based elements reported in stories (actors, activities and decisions) and official processes gives a ratio of 1.11 , which suggests that stories primarily convey contextual knowledge.

The ratio between context-based elements in stories and model-based elements in official processes is 2.25. This ratio provides a rough estimate of the amount of contextual knowledge that is often neglected by organizations when creating official processes.

Reflecting about the gathered contextual knowledge, we observe that elements related to time, people and individual decisions were predominant. Looking carefully into the collected data, we found that more people were usually involved in business processes than reported in official processes. We also found more time dependencies and more decisions than reported in official processes. It seems intriguing that even though time, people and decisions are fundamental concerns when specifying model-based behavior, they are also major concerns in the contextual dimension. We suggest that models describing business processes should better express the richness and openness of work in organizations in these three dimensions.

From the data gathered in the two cases, we note the participants reported a moderate amount of contextual knowledge about methods. Maybe the participants do not want to rationalize organizational work, or storytelling is inadequate to rationalize organizational work. Storytelling may promote telling how work is done instead of reflecting on how or why it should be done. Nevertheless, it seems relevant that the method still gathers a reasonable amount of contextual knowledge in the method category $(12.58 \%)$. The method may still be relevant to collect insightful knowledge about how work should be done.

We observed a lack of importance given by the participants to environmental factors and locations. However, this may be related to the specific nature of the two cases that were researched. Other organizations operating in more permeating and mobile scenarios may provide different results.

Our expectations regarding the expression of emotion were high at the beginning of the research. We expected stories to be full of emotional contents. However, the presence of elements in that category in the data was relatively low: they corresponded to $7.24 \%$ of the reported contextual knowledge. It was nevertheless interesting to realize that the majority of emotions were related to sensitive areas in business processes, where official processes conflicted with the attitudes and expectations of the participants. In particular, most of the negative emotions in the HEC and GP cases were time-related (waiting for something to happen), and some of the negative emotions reported in the HEC case were feedback-related (not knowing what was going on). We therefore suggest that emotional knowledge can be useful to optimize business processes from a usability perspective, addressing in particular awareness and feedback.

We found the individual stories provided by the participants had very significant contextual variability and diversity. Some participants were very concise in their stories. The most concise participant (GP3) provided only 34 elements, while the most expressive (GP12) provided 174. We find this wide range in storytelling capacity very interesting. Besides 
documenting business processes from different points of view, and perhaps different needs, the whole collection of stories contributes to develop an ecology of work patterns, which may better account for the diversity of organizational experiences faced by the stakeholders.

Contrasting these results with the internal and external categories of context discussed in Section 2, we observe that the gathered stories emphasized internal context. We cannot however discard the applicability of the method to analyze external context. Since the processes selected for this study had a strong emphasis on internal procedures, it seems reasonable that stories emphasize internal factors such as time, people, and interactional and decisional elements. Future research should apply the method to processes more dependent on external factors, e.g. sales, customer care and interorganizational processes.

\section{Conclusions}

The move from the flexible organization towards the knowledge organization requires full integration of model-based and context-based behaviors. In particular, it requires analyzing how contextual factors opportunistically influence and are influenced by participation in business activities, both planned and unplanned; and it also requires designing processes that articulate the two dimensions. The method discussed in this paper supports the elicitation and analysis of knowledge elements related to business processes. The application of the method in two cases suggests it is particularly adequate to elicit contextual elements related to time, people and decisions. To a lesser extent but nevertheless relevant, the method also elicits contextual elements related to methods, interactions, environment, and locations. Finally, the method also elicits elements related to activities, actors and decisions. Therefore, we suggest the model contributes to analyze integrated context/model behaviors is business processes.

A unique aspect of the method is using storytelling theory to capture knowledge about business processes. Storytelling theory provides the conceptual foundation to elicit traditional procedural knowledge, i.e. activities and decisions, fully integrated with contextual knowledge. The method then helps analyzing how context relates to model-based behavior. Furthermore, considering the ratio between the collected elements and the models documenting official processes, and the ratio between the context-based and model-based elements, we suggest the method is particularly effective to elicit process knowledge. Therefore, it seems the method should be added to the knowledge managers' tool set [18]. Of course, the method acceptance and use by knowledge managers, as well as its impact in organizations, remains to be investigated.

\section{References}

[1] Andrade, E., Van Der Aa, H., Leopold, H., Alter, S., and Reijers, H., "Factors Leading to Business Process Noncompliance and Its Positive and Negative Effects: Empirical Insights from a Case Study", Twenty-second Americas Conference on Information Systems, 2016.

[2] Antunes, P., "BPM and Exception Handling: Focus on Organizational Resilience", IEEE Transactions on System, Man, and Cybernetics Part C: Applications and Reviews, 41(3), 2011, pp. 383-392.

[3] Antunes, P., and Mourão, H., "Resilient Business Process Management: Framework and Services", Expert Systems with Applications, 38(2), 2011, pp. 1241-1254.

[4] Antunes, P., Simões, D., Carriço, L., and Pino, J., "An EndUser Approach to Business Process Modeling", Journal of Network and Computer Applications, 36(6), 2013, pp. 14661479.

[5] Argote, L., and Guo, J.M., "Routines and Transactive Memory Systems: Creating, Coordinating, Retaining, and Transferring Knowledge in Organizations", Research in Organizational Behavior, 36(65-84), 2016.

[6] Bacharach, S., and Aiken, M., "Communication in Administrative Bureaucracies", Academy of Management Journal, 20(3), 1977, pp. 365-377.

[7] Barker, R., and Gower, K., "Strategic Application of Storytelling in Organizations: Toward Effective Communication in a Diverse World", The Journal of Business Communication, 47(3), 2010, pp. 295-312.

[8] Bertelsen, O., "Design Artefacts: Towards a DesignOriented Epistemology", Scandinavian Journal of Information Systems, 12(1), 2000, pp. 2.

[9] Böhringer, M., "Emergent Case Management for Ad-Hoc Processes: A Solution Based on Microblogging and Activity Streams": Business Process Management Workshops, Springer, Heidelberg, 2011, pp. 384-395.

[10] Boyce, M., "Organizational Story and Storytelling: A Critical Review", Journal of Organizational Change Management, 9(5), 1996, pp. 5-26.

[11] Brézillon, P., "Focusing on Context in Human-Centered Computing", Ieee Intelligent Systems, 18(3), 2003, pp. 62-66. [12] Cabitza, F., and Simone, C., "Computational Coordination Mechanisms: A Tale of a Struggle for Flexibility", Computer Supported Cooperative Work, 22(4-6), 2013, pp. 475-529.

[13] Cecez-Kecmanovic, D., Galliers, R., Henfridsson, O., Newell, S., and Vidgen, R., "The Sociomateriality of Information Systems: Current Status, Future Directions", MIS Quarterly, 38(3), 2014, pp. 809-830.

[14] Dey, A., "Understanding and Using Context", Personal and Ubiquitous Computing, 5(1), 2001, pp. 4-7.

[15] Dumas, M., La Rosa, M., Mendling, J., and Reijers, H., Fundamentals of Business Process Management, Springer, Heidelberg, 2013.

[16] Elo, S., Kääriäinen, M., Kanste, O., Pölkki, T., Utriainen, K., and Kyngäs, H., "Qualitative Content Analysis: A Focus on Trustworthiness", Sage Open, 4(1), 2014. 
[17] Fisher, W., "Narration as a Human Communication Paradigm: The Case of Public Moral Argument", Communications Monographs, 51(1), 1984, pp. 1-22.

[18] Gavrilova, T., and Andreeva, T., "Knowledge Elicitation Techniques in a Knowledge Management Context", Journal of Knowledge Management, 16(4), 2012, pp. 523-537.

[19] Gerring, J., Case Study Research Principles and Practices, Cambridge University Press, Cambridge, 2007.

[20] Gregor, S., and Jones, D., "The Anatomy of a Design Theory", Journal of the Association of Information Systems, 8(5), 2007, pp. 312-335.

[21] Hevner, A., March, S., Park, J., and Ram, S., "Design Science in Information Systems Research", Management Information Systems Quarterly, 28(1), 2004, pp. 75-105.

[22] Kannengiesser, U., Neubauer, M., Krenn, F., Di Francescomarino, C., Majoe, D., and Bonaldi, D., "Towards Context-Aware Processes in People-Centered Factories of the Future", Proceedings of the 7th International Conference on Subject-Oriented Business Process Management, 2015.

[23] Kannengiesser, U., Totter, A., and Bonaldi, D., "An Interactional View of Context in Business Processes": S-BPM One Application Studies, 2014, pp. 42-54.

[24] Krumeich, J., Weis, B., Werth, D., and Loos, P., "EventDriven Business Process Management: Where Are We Now? A Comprehensive Synthesis and Analysis of Literature", Business Process Management Journal, 20(4), 2014, pp. 615633.

[25] Krylova, K., Vera, D., and Crossan, M., "Knowledge Transfer in Knowledge-Intensive Organizations: The Crucial Role of Improvisation in Transferring and Protecting Knowledge", Journal of Knowledge Management, 20(5), 2016, pp. 1045-1064.

[26] Kumar, R., Research Methodology, Pearson, Australia, 2005.

[27] Kurz, M., Fleischmann, A., Lederer, M., and Huber, S., "Planning for the Unexpected: Exception Handling and Bpm", International Conference on Subject-Oriented Business Process Management, 2013, pp. 123-149.

[28] Linhares, G., Borges, M., and Antunes, P., "Collaboration and Conflict in Software Review Meetings", International Journal of Information Technology \& Decision Making, 11(6), 2012, pp. 1065-1085.

[29] Mendonça, D., Jefferson, T., and Harrald, J., "Collaborative Adhocracies and Mix-and-Match Technologies in Emergency Management", Communications of the ACM, 50(3), 2007, pp. 44-49.

[30] Miles, M., and Huberman, A., Qualitative Data Analysis, SAGE, Thousand Oaks, California, 1994.

[31] Motahari-Nezhad, H., and Swenson, K., "Adaptive Case Management: Overview and Research Challenges", IEEE 15th Conference on Business Informatics, 2013, pp. 264-269.

[32] Orlikowski, W., and Gash, D., "Technological Frames: Making Sense of Information Technology in Organizations", ACM transactions on information systems, 12(2), 1994, pp. 174-207.

[33] Ploesser, K., Peleg, M., Soffer, P., Rosemann, M., and Recker, J., "Learning from Context to Improve Business Processes", BPTrends, 6(1), 2009, pp. 1-7.

[34] Polyvyanyy, A., Smirnov, S., and Weske, M., "Business Process Model Abstraction": Handbook on Business Process
Management 1, Springer, Berlin, Heidelberg, 2015, pp. 147165.

[35] Pratono, A., "Strategic Orientation and Information Technological Turbulence: Contingency Perspective in Smes", Business Process Management Journal, 22(2), 2016, pp. 368-382.

[36] Prusak, L., Groh, K., Denning, S., and Seely, J., Storytelling in Organizations, Routledge, London, 2004.

[37] Reichert, M., and Weber, B., Enabling Flexibility in Process-Aware Information Systems: Challenges, Methods, Technologies, Springer, Heidelberg, 2012.

[38] Rocha, R., Fantinato, M., Thom, L., and Eler, M., "Dynamic Product Line for Business Process Management", Business Process Management Journal, 21(6), 2015, pp. 12241256.

[39] Rosemann, M., and Recker, J., "Context-Aware Process Design: Exploring the Extrinsic Drivers for Process Flexibility", The 18th International Conference on Advanced Information Systems Engineering, 2006, pp. 149-158.

[40] Rotaru, K., Wilkin, C., and Churilov, L., "Proactive Risk Identification in Cross-Organizational Business Processes", 25th Australasian Conference on Information Systems, 2014. [41] Saidani, O., and Nurcan, S., "Towards Context Aware Business Process Modelling", 8th Workshop on Business Process Modeling, Development, and Support 2007.

[42] Seidel, S., Shortland, K., and Elzinga, D., "CreativityAware Business Process Management: What We Can Learn from Film and Visual Effects Production": Handbook on Business Process Management 2, Springer, Berlin Heidelberg, 2015, pp. 715-739.

[43] Simões, D., Antunes, P., and Cranefield, J., "Enriching Knowledge in Business Process Modelling: A Storytelling Approach", in (Razmerita, L., Phillips-Wren, G., and Jain, L., eds.): Innovations in Knowledge Management: The Impact of Social Media, Semantic Web and Cloud Computing, Springer, Heidelberg, 2016, pp. 241-267.

[44] Snowden, D., ..,(), Pp.. "Complex Acts of Knowing: Paradox and Descriptive Self-Awareness", Journal of Knowledge Management, 6(2), 2002, pp. 100-111.

[45] Snowden, D., and Boone, M., "A Leader's Framework for Decision Making", Harvard Business Review, November, 2007, pp. 69-76.

[46] Suchman, L., Plans and Situated Actions: The Problem of Human-Machine Communication, Cambridge University Press, Cambridge, UK, 1987.

[47] Van Der Aalst, W., "Business Process Management: A Comprehensive Survey", ISRN Software Engineering, 2013.

[48] Vom Brocke, J., Zelt, S., and Schmiedel, T., "On the Role of Context in Business Process Management", International Journal of Information Management, 36(3), 2016, pp. 486495. 